CYTOSKELETON

\section{A star is born}

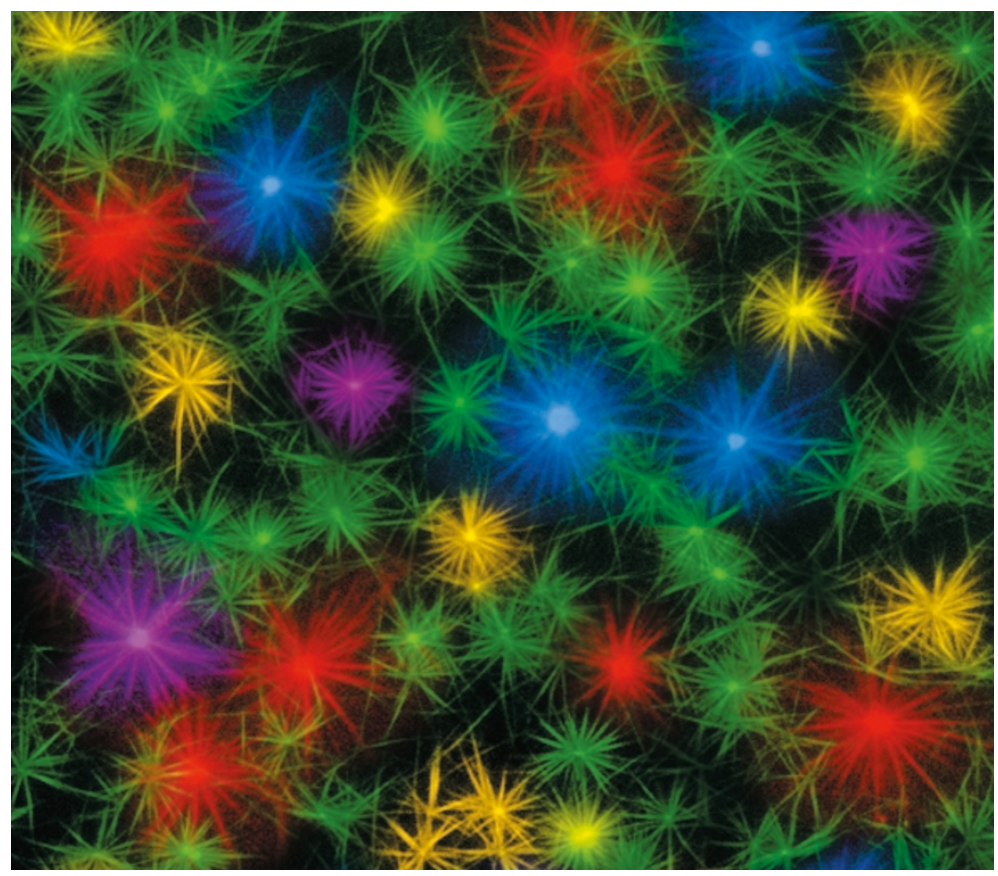

Image courtesy of D. Vignjevic et al., Northwestern Medical School, Chicago, USA.
Actin-rich finger-like filopodia let cells explore their environment, but little is known about how filopodial formation is initiated. The lack of an in vitro system for producing filopodia-like bundles has certainly been a handicap, so the development of such a system by Vignjevic et al., reported in The Journal of Cell Biology, is a welcome step. Using this system, the authors propose that filopodia form by the elongation of a pre-existing actin filament network by inhibition of capping and the subsequent bundling of these filaments.

The authors coated beads with Wiskott-Aldrich syndrome protein (WASP), a protein that facilitates filopodia formation, introduced them with actin into a rat brain extract, and visualized the resultant actin structures. Depending on their position under the coverslip, the structures were very different. 'Stars' of straight actin bundles emanating from the beads formed at the edge, whereas 'clouds' of actin filaments, or 'comets', formed in the centre. Vignjevic et al. reasoned that one or more proteins became depleted during sample spreading by adsorption to the glass coverslip. And indeed, reducing the concentration of some factor(s) in the extract - by glass depletion or fivefold dilution resulted in uniform star formation.

The stars' radial actin bundles resembled filopodia, so Vignjevic et al. looked for other similarities. Kinetic and structural analysis of star formation showed that close to the bead was a dendritic network (resembling lamellipodia), from which long, unbranched filaments emanated and merged into bundles (resembling filopodia) further away. There were also differences in the localization of proteins associated with lamellipodia or filopodia, such as the actin-related protein 2/3 (Arp 2/3) complex. Arp2/3 was present in the dendritic network, but not in bundles.

WASP-family members activate the Arp 2/3 complex, which nucleates actin filaments. Depleting Arp $2 / 3$ abolished actin assembly around beads, but star formation

\section{Preventing a repeat}

Replication is limited to a single round per cell cycle, and re-replication in metazoans is prevented by a regulatory mechanism that involves Cdk2 kinase. Ryuji Yamaguchi and John Newport now report in Cell the identification of other components of this Cdk2-dependent mechanism.

Yamaguchi and Newport noticed that, when they added sperm chromatin to Xenopus egg cytosol, pre-replication complexes (pre-RCs) assembled rapidly onto sperm DNA. By contrast, when adding sperm chromatin to nuclear extract, MCM helicase - an initiation factor and component of the pre- $\mathrm{RC}$ - failed to bind to the chromatin. As nuclear extract contains a high concentration of Ran-GTP compared with the cytosol, and given its many functions in nuclear processes, the authors examined whether Ran-GTP is responsible for preventing assembly of MCM into preRCs. They showed that addition to nuclear extract of a mutant Ran protein, RanT24N, which binds weakly to GDP and not to GTP, rescued MCM assembly into pre-RCs, whereas wild-type Ran did not.

Next, the authors wanted to know whether RanT24N had the same effect in intact nuclei. As expected, MCM binding was inhibited in pre-formed nuclei that were supplemented with cytosol containing MCM, and indeed, this inhibition could be reversed by addition of RanT24N. Moreover, in the presence of RanT24N, replication was faster and continued for longer, and bromodeoxyuridine (BrdU) incorporation confirmed that RanT24N induced multiple rounds of replication. When RanT24N was added to reconstituted nuclei immediately after replication (G2 nuclei), MCM re-associated with the chromatin and replication resumed.

By increasing the low cytosolic concentration of Ran-GTP, binding of MCM to chromatin was reduced. Moreover, in the presence of a Ran mutant, RanQ69L which can bind but not hydrolyse GTP MCM loading was completely blocked. Yamaguchi and Newport postulated that the low Ran-GTP concentration in the cytosol allows MCM to assemble into pre-RCs prior to nuclear assembly.

Because the nuclear Ran-GTP level is constantly high, the authors hypothesized that Ran-GTP must link to another regulatory component to allow pre-RC formation during G1. Cdk2 kinase is a likely candidate, as it is known to be essential for inhibiting re-replication. When Cdk2 was inactivated by Cdk2 inhibitor p27Kip1, MCM loading was no longer inhibited by RanQ69L. So, Ran-GTP-induced MCM inhibition is dependent on Cdk2.

Using an anti-MCM7 antibody, Yamaguchi and Newport immunoprecipitated the nuclear-export protein exportin-1/Crm1. Moreover, increasing the Ran-GTP concentration by addition of RanQ69L increased the association of $\mathrm{Crm} 1$ with MCM. By contrast, addition of RanT24N destabilized the Crm1-MCM complex. Immunodepletion of $\mathrm{Crm} 1$ meant that RanQ69L could no longer inhibit MCM assembly, and inhibition could be restored by adding Crml back to the extract.

So, the authors have identified yet another nuclear function for the small GTPase Ran, and unravelled one of possibly several (redundant) mechanisms for preventing re-replication in metazoans.

Arianne Heinrichs

\section{(2) References and links}

ORIGINAL RESEARCH PAPER Yamaguchi, R. \& Newport, J. A role for Ran-GTP and Crm1 in blocking re-replication. Cell 113, 115-125 (2003) 
was restored when $\operatorname{Arp} 2 / 3$ was added back, indicating that Arp2/3 mediates star formation. Furthermore, the adsorption/dilution experiments had hinted that a decreased amount of factor(s) in the extract were crucial in star formation. Increased levels of a candidate factor, capping protein, antagonized star formation. Decreased levels (for example, at the edge of the coverslip) probably mediate actin-filament elongation.

On the basis of their results, Vignjevic et al. attempted reconstitution experiments using WASPcoated beads, actin and Arp2/3, but found that they had to add an actin-bundling protein, fascin, to promote star formation. So, the authors propose three steps nucleation (by Arp2/3), elongation (by decreased capping protein) and bundling (by fascin) — for star, or filopodia, formation.

Katrin Bussell

di) References and links ORIGINAL RESEARCH PAPER Vignjevic, D. et al. Formation of filopodia-like bundles in vitro from a dendritic network. J. Cell Biol. 160 951-962 (2003)

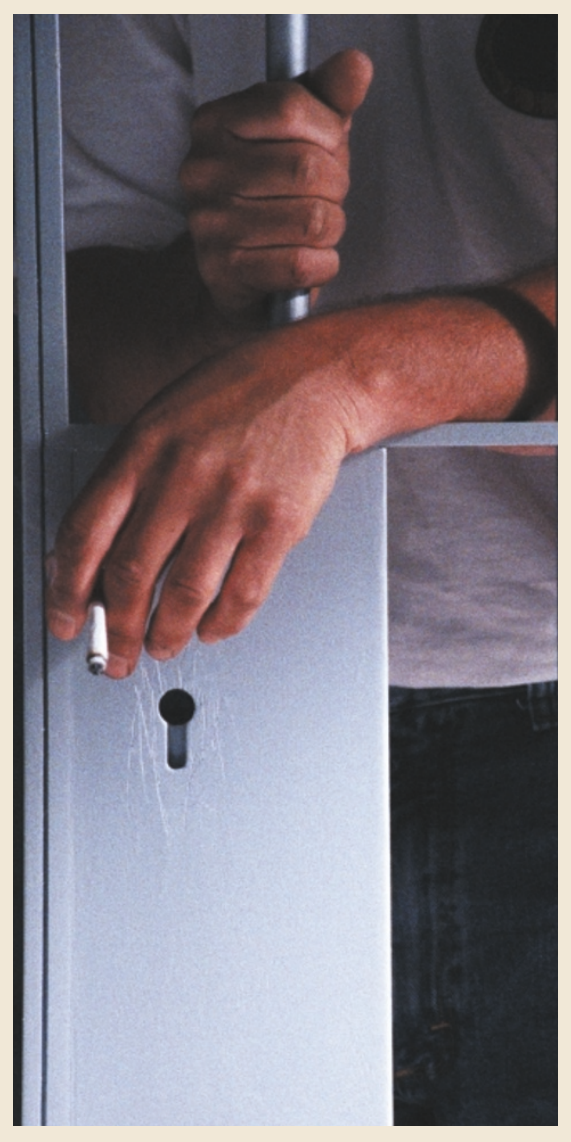

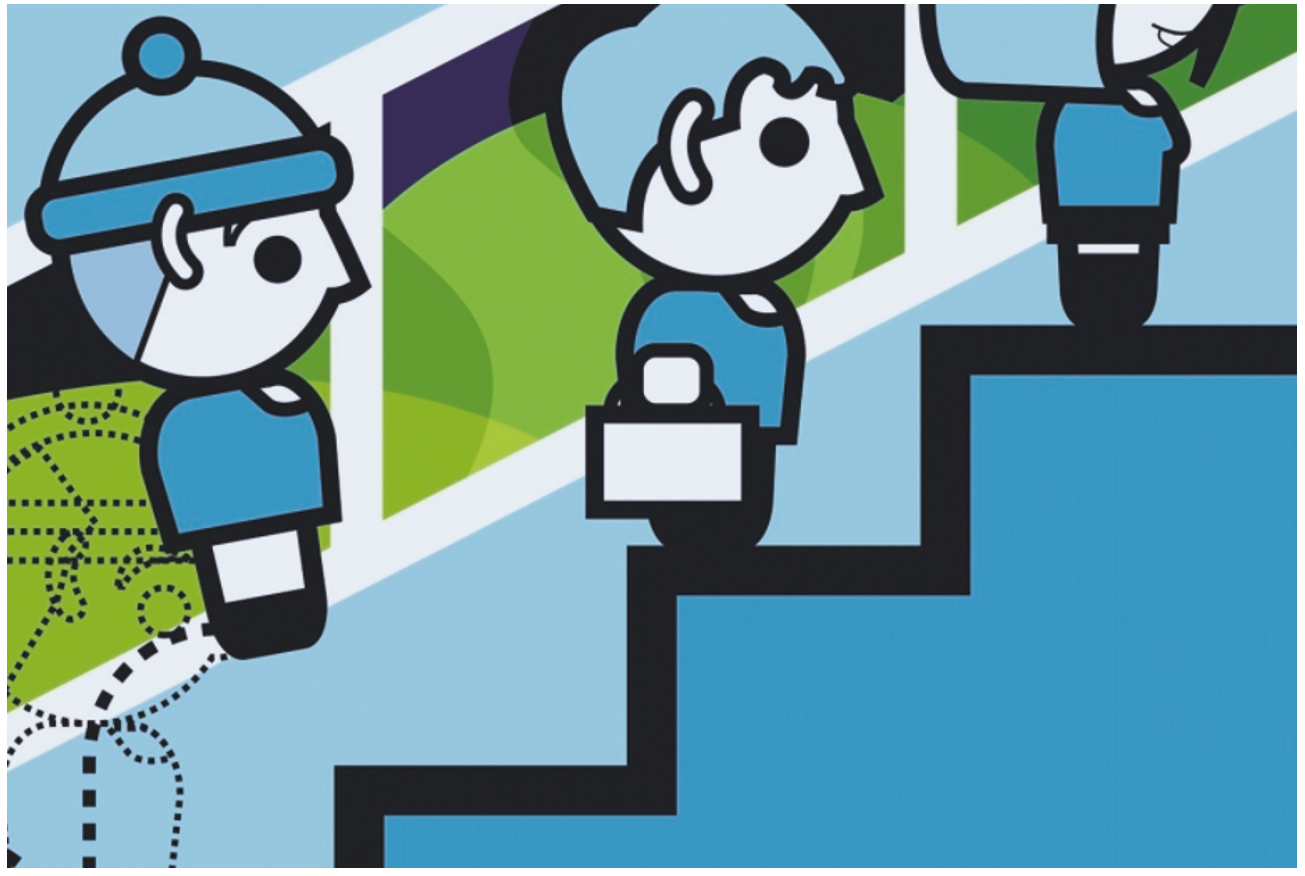

\section{PROTEIN TRANSLOCATION}

\section{Doing the three-step}

The TIM22 complex - a translocase of the inner mitochondrial membrane - is responsible for inserting precursor proteins that contain several transmembrane segments and internal signal peptides (for example, metabolite carriers) into the inner mitochondrial membrane. To do this, TIM22 uses the membrane potential $(\Delta \psi)$ as its only external energy source, but exactly how does this complex fulfil its role? Now, in Science, Nikolaus Pfanner and colleagues provide new insights.

A complete, functional TIM22 complex had never before been purified, so the authors first developed a method to isolate the entire, functional, yeast TIM22 complex. Once purified, they reconstituted the complex into liposomes and fused them with a planar lipid bilayer. They then monitored the changes in current recordings that occurred on addition of an internal signal peptide (P2) from the phosphate carrier.

Purified Tim22 - an integral membrane subunit of the TIM22 complex - has previously been shown to form a single voltage-activated pore, which 'flickers' on addition of $\mathrm{P} 2$ at a $\Delta \psi$ greater than $140 \mathrm{mV}$. By contrast, Pfanner and colleagues found that the TIM22 complex has two tightly coupled pores. When P2 was added to TIM22, they found that the flickering/gating occurred in only one of the two pores (the other pore was mainly closed), and that this gating was more rapid than for Tim 22 alone, and could be induced at a lower $\Delta \psi(75 \mathrm{mV})$.

So, how does this information relate to the natural process in organello? Mitochondrial import of carrier proteins occurs in five stages, and stage IV - insertion into the inner membrane - has remained poorly understood. Pfanner and coworkers therefore monitored the effects of lowering $\Delta \psi$, using the protonophore CCCP, on the late stages of import of both the phosphate carrier and the dicarboxylate carrier.

At high $\Delta \psi$ (no CCCP), both carriers were mainly present in their mature, dimeric form (stage $\mathrm{V}$ ), whereas, in the absence of $\Delta \psi$ (high CCCP levels), they were present in the lower-molecularweight stage III form (that is, they are probably associated with small, soluble Tim proteins in the intramembrane space). However, at intermediate $\Delta \psi$ values $(<60 \mathrm{mV})$, the authors could observe a stage IV intermediate - a TIM22-carrier complex. For both carriers, restoring $\Delta \psi$ resulted in stage $\mathrm{V}$ being reached, which indicates that this stage IV intermediate represents a productive translocation intermediate.

However, Pfanner and colleagues further noticed that a small, but significant, carrier fraction associated with TIM 22 in the absence of $\Delta \psi$, and they proposed that this fraction might represent a 'tethered' form of the carrier, rather than the 'docked' stage IV intermediate described above. Indeed, they showed that the tethered intermediate was more resistant to ionic-strength increases than the docked intermediate, which indicates that the "...tethered and docked forms of the carrier reside in different molecular environments at the TIM22 complex, the tethered protein probably being in a more hydrophobic environment".

The twin-pore TIM22 complex therefore inserts precursors into the inner mitochondrial membrane in three steps. First, the precursor is tethered to TIM22 in a $\Delta \psi$-independent step. Then, in the first voltage-dependent step, the precursor docks in TIM22 (a $\Delta \psi$ below that needed to influence pore activity was sufficient for this step, which indicates that $\Delta \psi$ acts directly on the precursor). Finally, in the second voltage-dependent step, a $\Delta \psi$ greater than $\sim 70 \mathrm{mV}$ and the presence of a signal peptide activate pore gating to complete the membraneinsertion process.

Rachel Smallridge

(2) References and links

ORIGINAL RESEARCH PAPER Rehling, P. et al. Protein insertion into the mitochondrial inner membrane by a twin-pore translocase. Science 299, 1747-1751 (2003) 